\title{
VRN1 genes variability in tetraploid wheat species with a spring growth habit
}

\author{
Irina Konopatskaia ${ }^{12^{*}}$, Valeriya Vavilova', Elena Ya. Kondratenko ${ }^{1}$, Alexandr Blinov ${ }^{1}$ and Nikolay P. Goncharov ${ }^{1,3}$
}

From The International Conference on Bioinformatics of Genome Regulation and StructurelSystems Biology (BGRSISB-2016)

Novosibirsk, Russia. 29 August-2 September 2016

\begin{abstract}
Background: Vernalization genes VRN1 play a major role in the transition from vegetative to reproductive growth in wheat. In di-, tetra- and hexaploid wheats the presence of a dominant allele of at least one VRN1 gene homologue (Vrn-A $1, V r n-B 1, V r n-G 1$ or Vrn-D1) determines the spring growth habit. Allelic variation between the Vrn-1 and vrn-1 alleles relies on mutations in the promoter region or the first intron. The origin and variability of the dominant VRN1 alleles, determining the spring growth habit in tetraploid wheat species have been poorly studied.
\end{abstract}

Results: Here we analyzed the growth habit of 228 tetraploid wheat species accessions and $25 \%$ of them were spring type. We analyzed the promoter and first intron regions of VRN1 genes in 57 spring accessions of tetraploid wheats.

The spring growth habit of most studied spring accessions was determined by previously identified dominant alleles of VRN1 genes. Genetic experiments proof the dominant inheritance of Vrn-A1d allele which was widely distributed across the accessions of Triticum dicoccoides. Two novel alleles were discovered and designated as Vrn-A1b.7 and Vrn-BIdic. Vrn-A 16.7 had deletions of $20 \mathrm{bp}$ located $137 \mathrm{bp}$ upstream of the start codon and mutations within the VRN-box when compared to the recessive allele of $v r n-A 1$. So far the Vrn-A1d allele was identified only in spring accessions of the $T$. dicoccoides and T. turgidum species. Vrn-B1dic was identified in T. dicoccoides IG46225 and had $11 \%$ sequence dissimilarity

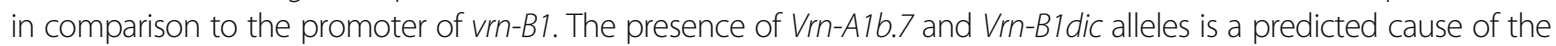
spring growth habit of studied accessions of tetraploid species. Three spring accessions T. aethiopicum K-19059, T. turanicum K-31693 and T. turgidum cv. Blancal possess recessive alleles of both VRN-A1 and VRN-B1 genes. Further investigations are required to determine the source of spring growth habit of these accessions.

Conclusions: New allelic variants of the VRN-A1 and VRN-B1 genes were identified in spring accessions of tetraploid wheats. The origin and evolution of VRN-A1 alleles in di- and tetraploid wheat species was discussed.

Keywords: Evolution, Growth habit, Triticum, vernalization, VRN1 gene, Wheat

\section{Background}

Flowering time is a critical agronomical trait that has a major impact on the adaptation to local climate and environmental conditions and grain yield in wheat species. Wheat cultivars differ in their requirements for extended exposure to low-temperature (vernalization) to initiate the transition from vegetative growth to flowering [1].

\footnotetext{
* Correspondence: sormacheva@bionet.nsc.ru

'The Federal Research Center Institute of Cytology and Genetics SB RAS,

Prospekt Lavrentyeva, 10, Novosibirsk 630090, Russian Federation

${ }^{2}$ Novosibirsk State University, Pirogova 2, 630090 Novosibirsk, Russian

Federation

Full list of author information is available at the end of the article
}

Ancestors of wheat, as well as modern wheat species with a winter growth habit, are planted in autumn and flower during the subsequent spring. These species require vernalization for transition from vegetative to reproductive growth. The vernalization requirement prevents the fragile flower meristems from being damaged by low temperatures and ensures that flowering occurs under optimal conditions in spring. Modern wheat cultivars with spring growth habit lack this vernalization requirement and can be planted in spring [2].

Vernalization-induced flowering in wheat is mainly controlled by the vernalization genes VRN1, VRN2, VRN3 and VRN4 which interact with each other as well 
as other flowering control pathways [1-8]. VRN1 genes mapped to the long arms of the 5 homoeological group chromosomes play a central role in complex vernalization pathways [9-12]. The floral activator VRN1 encodes a MADS-box transcription factor that is required for the initiation of reproductive development at the shoot apical meristem [3, 13, 14]. The expression of $V R N 1$ occurs at a low basal level but a measurable increase is seen during prolonged treatment with low temperatures $[3,13,14]$.

In di-, tetra- and hexaploid wheats the presence of a dominant allele of at least one VRN1 gene homologue (Vrn-A1, Vrn-B1, Vrn-G1 or Vrn-D1) determines the spring growth habit. Allelic variation between the $\mathrm{Vrn}-1$ and vrn-1 alleles relies on mutations in the promoter region or the first intron.

Analysis of diploid wheat species revealed three recessive alleles vrn- $A^{m} 1, v r n-A 1 u$ and $v r n-A^{m} 1 b$ which determine the winter growth habit $[3,15-18]$. The vrn- $A^{m} 1$ allele is distributed in all diploid wheat species and represents the only allelic variant so far identified in Triticum sinskajae A. Filat. et Kurk. [3, 15-18]. The vrn-A1u of T. urartu Thum. ex Gandil. is identical to vrn-A1 of winter accessions of polyploid wheats and differs from vrn- $A^{m} 1$ by a short deletion in the promoter $[16,17]$. The vrn$A^{m} 1 b$ with a 48-bp deletion in the VRN1 promoter in compare to $v r n-A^{m} 1$ so far was found only in accessions of T. monococcum L. $[3,18]$. In diploid wheat $T$. monococcum several dominant $\mathrm{Vrn}-\mathrm{Al}$ alleles which possess variable mutations in the promoter and/or first intron region were identified [3, 15-18]. Two dominant alleles from diploid wild wheat $T$. boeoticum Boiss. $V r n-A^{m} 1 f$ and Vrn- $A^{m} 1 a(V r n-A 1 h)$ posses short deletions in the promoter region $[16,17]$. No dominant alleles were identified in $T$. urartu $[16,17]$.

In tetraploid wheat species of sections Timopheevii A. Filat. et Dorof. and Dicoccoides Flaksb. five recessive alleles of VRN1 genes (vrn-A1(vrn-A1u), vrn-A1b.3, vrn$A 1 b .4, v r n-B 1$ and $v r n-G 1)$ were identified $[16,17,19]$. The sequences of $v r n-B 1$ and $v r n-G 1$ alleles were identical to each other [16]. Several dominant and recessive alleles of $V R N-A 1, V R N-B 1$ and $V R N-G 1$ genes were identified in tetraploid wheat of two sections Timopheevii A. Filat. et Dorof. and Dicoccoides Flaksb. [16, 17, 19-22]. Thus, analysis of tetraploid wheats T. timopheevii (Zhuk.) Zhuk. and $T$. araraticum Jakubz. revealed one dominant allele of the $V R N-A 1$ gene $(V r n-A 1 f)$ comprising two deletions in the promoter region, and the only dominant allele of VRN-G1 gene $(V r n-G 1 a)$ with the insertion in the promoter region [16]. Ten various dominant alleles of the $V R N-A 1$ gene which possess different mutations in compare to vrn-A1 allele were identified in different tetraploid wheat species of section Dicoccoides Flaksb. Seven of them possess deletions of variable length in the promoter $(V r n-A 1 b$ (Vrn-A1b.1), Vrn-A1b.2, Vrn-A1b.5, Vrn-A1b.6, Vrn-A1e,
Vrn-Alf and Vrn-A1d), one (Vrn-A1i) has nucleotide substitution and one (Vrn-Ala (Vrn-Ala.3)) has a foldback element insertion in the promoter region $[16,17,19,20]$. The first intron sequence of the VRN-A1 gene from $T$. durum Desf. cultivar Lebsock was found to contain a large deletion identical to T. durum 'Langdon' [21, 22]. Further analysis allowed to identify this allele in accessions of $T$. turgidum L., T. carthlicum Nevski, T. polonicum L., T. dicoccoides (Körn. ex Aschers. et Graebn.) Schweinf. and T. durum Desf. [17, 19]. Novel allelic variant VRN-A1f-like identified by Ivaničová et al. [23] possess mutations in both promoter and first intron regions of $V R N-A 1$, while only mutations within first intron is the reason of the spring growth habit of $T$. militinae accession.

In tetraploid wheats of the section Dicoccoides four dominant alleles of $V R N-B 1$ for which the variability is characterized by the mutations in the promoter region (insertion of repeated elements or short deletions) $[16,19,21]$. The $V r n-B 1 a$ is the only dominant allele with the large deletion in the first intron which was identified in the Dicoccoides accessions [19, 22]. The only dominant allele Vrn-G1a from section Timopheevii is characterized by foldback element insertion in the promoter region [16].

For a long time vrn-A1 was the only recessive allele identified in hexaploid wheat, but recently additional allele vrn-A1b.3 was identified in T. vavilovii (Thum.) Jakubz. and T. spelta L [19]. Seven dominant alleles of the VRN-A1 gene, Vrn-A1a.1, Vrn-A1a.2, Vrn-A1b (Vrn$A 1 b .1), V r n-A 1 b .2, V r n-A 1 b .6, V r n-A 1 c$ and $V r n-A 1 f$ were found in hexaploid wheat $[16,19,20,22,23]$. The majority of the spring cultivars carry a Vrn-Ala.1 allele that has a miniature inverted-repeat transposable element (MITE) insertion and duplication in the promoter region [19]. Vrn-A1b allelic variants and Vrn-A1f allele have mutations and deletions of variable lengths in the promoter region, whereas $V r n-A 1 c$ has a deletion in the first intron in comparison to the recessive vrn-A1 allele $[16,19,20,22,23]$. Most of the dominant alleles of $V R N-B 1$ and $V R N-D 1$ genes possess deletions in the first intron (Vrn-B1a, Vrn-B1b, Vrn-B1c and Vrn-D1a) [16, 19, 22, 24-27]. Several recently identified alleles (Vrn-B1ins, Vrn-D1c, Vrn-D1s) are characterized by different insertions within the promoter region $[19,28,29]$. The $V r n-D 1 b$ is characterized by the deletion in intron 1 identical to Vrn-D1a allele and a single nucleotide mutation at promoter and is associated with facultative growth habit [30].

The evolution of spring cultivars of wheats from winter ancestors is a key event in the post-domestication spread of wheat [1]. However, studies of the major vernalization gene VRN1 are mostly limited to the analysis of di- and hexaploid wheat species. In the present study we investigate the growth habit and variability of promoter and first intron regions of VRN1 genes in 
accessions of twelve tetraploid wheat species of sections Dicoccoides and Timopheevii.

\section{Methods \\ Plant material}

Accessions of 12 tetraploid wheat species were obtained from the following gene banks: N.I. Vavilov Institute of Plant Genetic Resources (VIR, Russian Federation), The Federal Research Center Institute of Cytology and Genetics SB RAS (Russian Federation), the National Small Grains Collection (NSGC, USA), International Center for Agricultural Research in the Dry Area (ICARDA, Syria), Kyoto University (Japan). Place of origin, specimen voucher and growth habit of each accession are presented in Additional file 1: Table S1.

\section{Greenhouse experiments}

The growth habit of tetraploid wheat species was evaluated by growing in the greenhouse at $20-25{ }^{\circ} \mathrm{C}$ under a long photoperiod (18 h light) without vernalization treatment. $\mathrm{F}_{1}$ hybrids of tetraploid near-isogenic line $T$. dicoccum Black Spring VRN-B1 Emmer (i: BS2E) and $T$. dicoccum (Schrank) Schuebl. cv. Black Winter Emmer (BWE) were used as controls. In i: BS2E, spring growth habit is determined by the dominant allele of the Vrn-B1 gene [31]. T. dicoccum $\mathrm{cv}$. BWE has a winter growth habit. $\mathrm{F}_{1}$ hybrids of NIL BS2E with $T$. dicoccum cv. BWE have the genotype $V r n-B 1 / v r n-B 1$ and represent the latest maturing spring form at the border between spring vs. winter phenotypes, according to Pugsley [32] and Goncharov [33]. Accessions that headed before $F_{1}$ hybrids were classified as spring, whereas accessions that remained in the vegetative phase were classified as winter. A detailed procedure is described in Goncharov [33].

To determine days to heading of spring accessions ten plants of each accession were grown in the greenhouse at $20-25{ }^{\circ} \mathrm{C}$ under a long photoperiod (18 h light) without vernalization treatment. Mean number of days to heading $(\mathrm{X} \pm s)$ and mean error $(s)$ were estimated using standard Microsoft office software.

The number of dominant $V R N$ genes in tetraploid wheat was identified based on the segregations in the $F_{2}$ generations. $F_{1}$ hybrids between six accessions of tetraploid wheats and three tester lines, winter accessions $T$. dicoccum $\mathrm{cv}$. BWE, T. dicoccum Black Spring VRN-A1 Emmer (i: BS1E) and i: BS2E, were produced by emasculation of mother's plant spikes and pollination with flowering father plant spikes using twirl-method. T. dicoccum cv. BWE has recessive $v r n-A 1$ and $v r n-B 1$ alleles while two near-isogenic test lines are characterized with the only specific dominant allele: $V r n-A 1$ in i: BS1E and $V r n-B 1$ of i: BS2E [32]. The segregation into spring versus winter forms for each cross was identified and compared with the expected segregation ratio using the Pierson chi-square test.

\section{Total DNA isolation, PCR amplification, cloning and} sequencing

Total DNA was isolated from $100 \mathrm{mg}$ of leaves using the DNeasy Plant Mini Kit (QIAGEN) according to the manufacturer's protocol. A set of primers were used to amplify the promoter and first intron sequences of $V R N-A 1, V R N$ $B 1$ and $V R N-G 1$ genes (Additional file 1: Table S2).

Polymerase chain reactions (PCR) were performed in a $20 \mu \mathrm{l}$ volume with $10 \mathrm{mM}$ Tris- $\mathrm{HCl}(\mathrm{pH} 8.9), 1 \mathrm{mM}$ $\left(\mathrm{NH}_{4}\right)_{2} \mathrm{SO}_{4}, 4 \mathrm{mM} \mathrm{MgCl} 2,200 \mu \mathrm{M}$ of each dNTP, $0.5 \mu \mathrm{M}$ of each primer, 1 unit of Taq DNA polymerase and $0.1 \mu \mathrm{g}$ of genomic DNA. The PCR program included an initial denaturation step for $3 \mathrm{~min}$ at $94{ }^{\circ} \mathrm{C}$ and 33 cycles of amplification consisting of $30 \mathrm{~s}$ denaturation at $94{ }^{\circ} \mathrm{C}, 40 \mathrm{~s}$ annealing at $52{ }^{\circ} \mathrm{C}$, and $1 \mathrm{~min}$ extension at $72{ }^{\circ} \mathrm{C}$. PCR products were separated by agarose gel electrophoresis and purified using a QIAquick Gel Extraction Kit (QIAGEN). Purified fragments were cloned into a pGEM ${ }^{\circ}$ - $\mathrm{T}$ Easy vector using a pGEM-T Easy kit (Promega) and amplified with M13 primers $\left(\mathrm{M} 13 \mathrm{~F}=5^{\prime}\right.$-GTTTTCCCAGTCACGAC-3 ${ }^{\prime}$, M13R $=5^{\prime}$-AGCGGATAACAATTTCACACAGGA-3'). Sequencing reactions were performed with $200 \mathrm{ng}$ of the product and ABI BigDye Terminator Kit on an ABI 3130XL Genetic Analyser (Applied Biosystems) in SB RAS Genomics Core Facility (http://www.niboch.nsc.ru/ doku.php/corefacility). In total 10 clones were sequenced for each target region of all wheat accessions with spring growth habit.

The sequences of the promoter and first intron region of VRN-A1, VRN-B1 and VRN-G1 genes were deposited in GenBank (accession numbers are given in Tables 1 and 2).

\section{Sequence analyses}

Nucleotide sequence alignments were performed using Vector NTI AdvanceTM version 10.0 program and improved with the MUSCLE algorithm in UGENE software (http://ugene.unipro.ru/) [34, 35].

\section{Results \\ Growth habit of tetraploid wheat species}

To analyze the variability of the promoter and first intron regions of $V R N-A 1, V R N-B 1$ and $V R N-G 1$ genes we chose a number of tetraploid wheat accessions, covering all species from both Timopheevii and Dicoccoides sections (Additional file 1: Table S1).

The growth habit of 228 accessions of tetraploid wheat species was checked by comparison to $F_{1}$ hybrids of i: BS2E and winter $T$. dicoccum $\mathrm{cv}$. BWE (see Methods section). In total, 57 accessions of 10 tetraploid wheat species headed before the hybrids and thus revealed a spring growth habit (Table 1; Additional file 1: Table S1). For each of 57 spring accessions 10 plants were grown under glasshouse conditions and days to heading were 


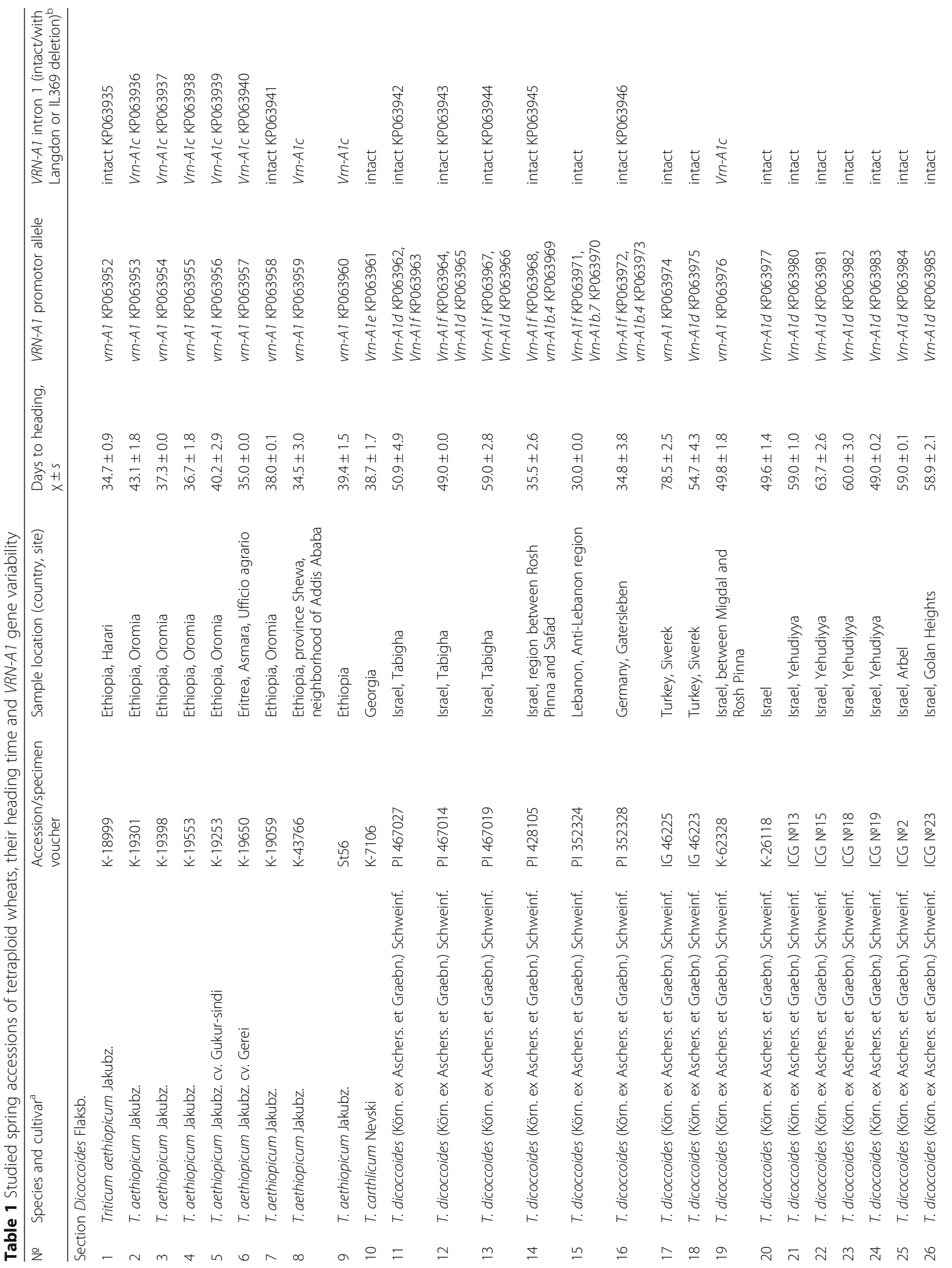




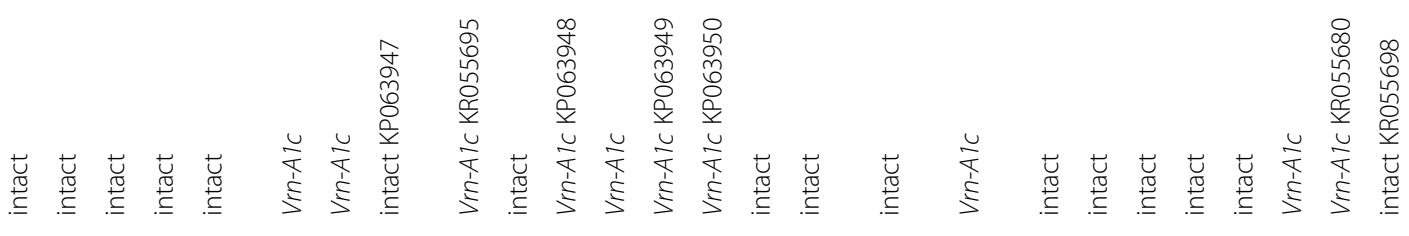

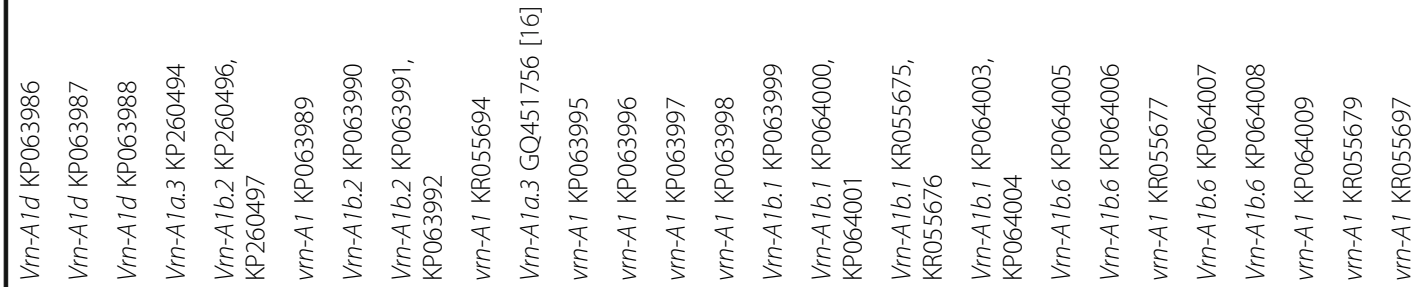

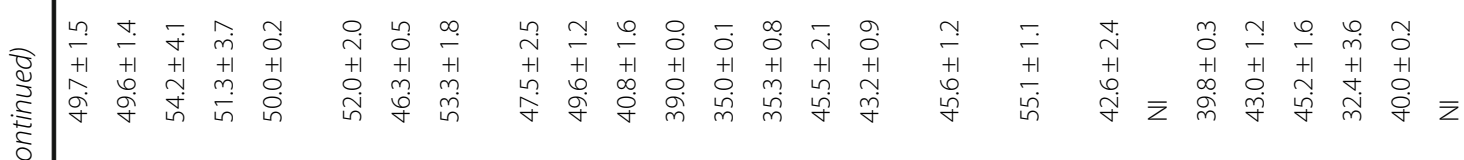

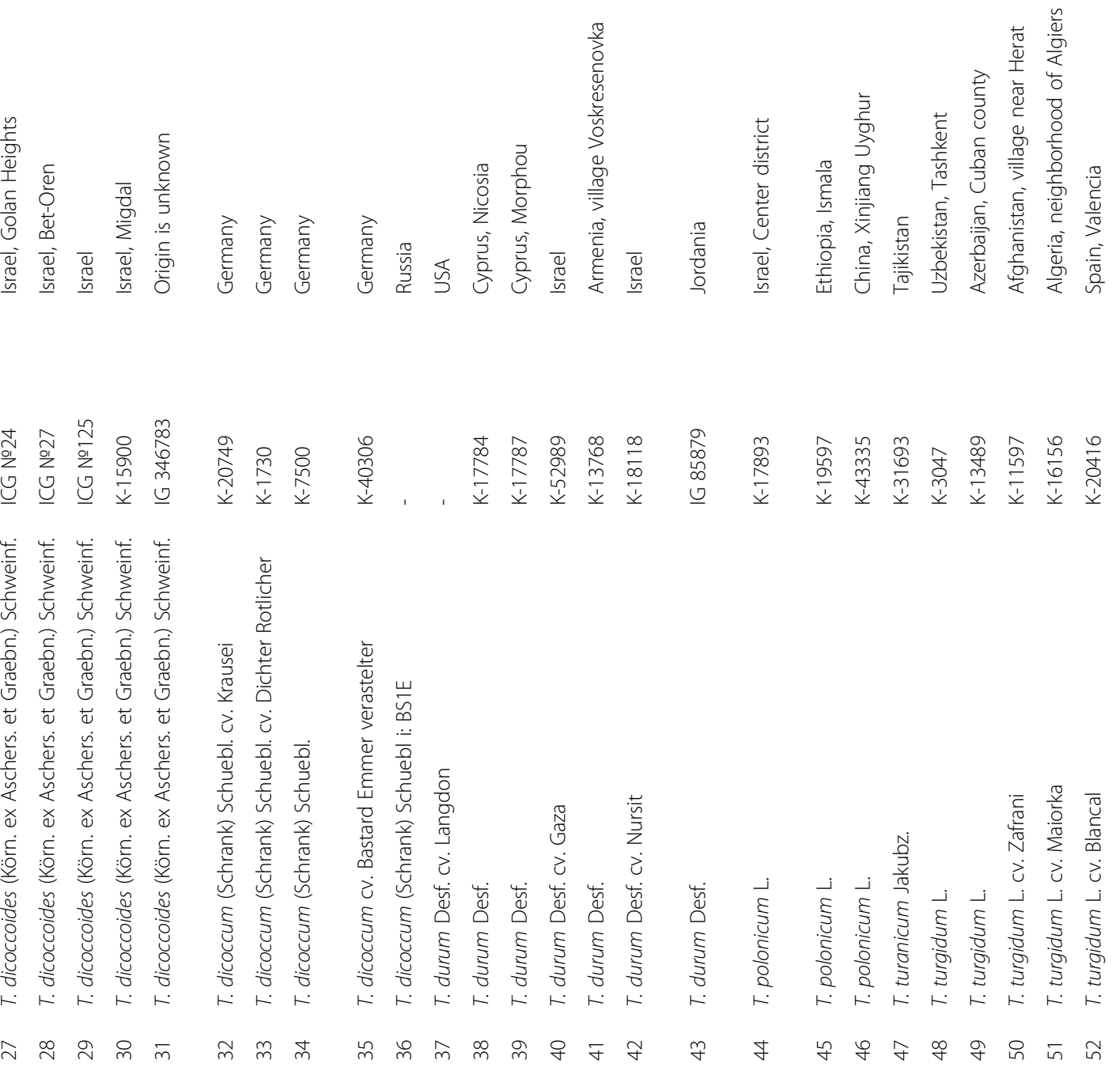


The Author(s) BMC Plant Biology 2016, 16(Suppl 3):244

Page 98 of 161

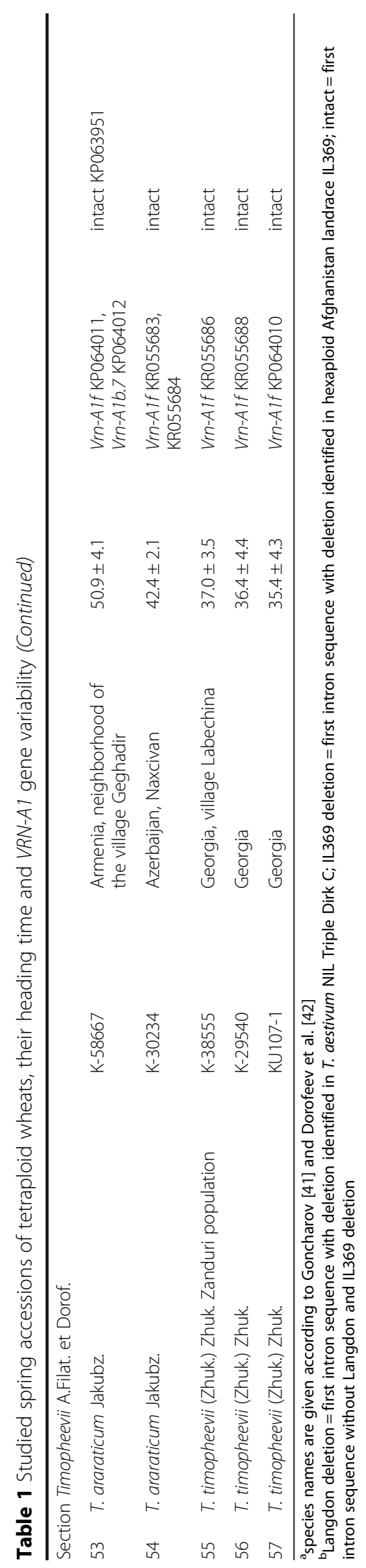


Table $2 V R N-B 1$ and $V R N-G 1$ genes variability in spring accessions of tetraploid wheats used in the study

\begin{tabular}{|c|c|c|c|c|c|c|}
\hline № & Species and cultivar ${ }^{\mathrm{a}}$ & $\begin{array}{l}\text { Accession/ } \\
\text { specimen } \\
\text { voucher }\end{array}$ & $\begin{array}{l}\text { Sample location } \\
\text { (country, site) }\end{array}$ & $\begin{array}{l}\text { Days to heading, } \\
x \pm s\end{array}$ & $\begin{array}{l}V R N-B 1 N R N-G 1 \\
\text { promotor allele }\end{array}$ & $\begin{array}{l}\text { VRN-B1NRN-G1 intron allele } \\
\text { (intact/with TDB deletion) }\end{array}$ \\
\hline \multicolumn{7}{|c|}{ Section Dicoccoides Flaksb. } \\
\hline 1 & T. aethiopicum Jakubz. & K-18999 & Ethiopia, Harari & $34.7 \pm 0.9$ & vrn-B1 KP063919 & Vrn-B1a KP063931 \\
\hline 2 & T. aethiopicum Jakubz. & K-19059 & Ethiopia, Oromia & $38.0 \pm 0.1$ & vrn-B1 KP063930 & intact \\
\hline 3 & $\begin{array}{l}\text { T. dicoccoides (Körn. } \\
\text { ex Aschers. et Graebn.) } \\
\text { Schweinf. }\end{array}$ & IG 46225 & Turkey, Siverek & $78.5 \pm 2.5$ & $\begin{array}{l}\text { vrn-B1 KP063925, } \\
\text { Vrn-B1dic } \\
\text { KP063926 }\end{array}$ & intact KP063932 \\
\hline 4 & T. turanicum Jakubz. & K-31693 & Tajikistan & $39.8 \pm 0.3$ & vrn-B1 KR055678 & intact \\
\hline 5 & T. turgidum L. Cv. Blancal & K-20416 & Spain, Valencia & $\mathrm{NI}$ & vrn-B1 KR055699 & intact \\
\hline \multicolumn{7}{|c|}{ Section Timopheevii A.Filat. et Dorof. } \\
\hline 6 & T. araraticum Jakubz. & K-58667 & $\begin{array}{l}\text { Armenia, neighborhood } \\
\text { of the village Geghadir }\end{array}$ & $50.9 \pm 4.1$ & vrn-G1 KR055682 & intact \\
\hline 7 & T. araraticum Jakubz. & K-30234 & Azerbaijan, Naxcivan & $42.4 \pm 2.1$ & $\begin{array}{l}\text { Vrn-Gla } \\
\text { KR055685 }\end{array}$ & intact \\
\hline 8 & $\begin{array}{l}\text { T. timopheevii (Zhuk.) Zhuk. Zanduri } \\
\text { population }\end{array}$ & K-38555 & $\begin{array}{l}\text { Georgia, village } \\
\text { Labechina }\end{array}$ & $37.0 \pm 3.5$ & $\begin{array}{l}\text { Vrn-Gla } \\
\text { KR055687 }\end{array}$ & intact \\
\hline 9 & T. timopheevii (Zhuk.) Zhuk. & K-29540 & Georgia & $36.4 \pm 4.4$ & $\begin{array}{l}\text { Vrn-G1a } \\
\text { KR055689 }\end{array}$ & intact \\
\hline 10 & T. timopheevii (Zhuk.) Zhuk. & KU107-1 & Georgia & $35.4 \pm 4.3$ & $\begin{array}{l}\text { Vrn-Gla } \\
\text { KR055690 }\end{array}$ & intact \\
\hline
\end{tabular}

${ }^{a}$ species names are given according to Goncharov [41] and Dorofeev et al. [42]

${ }^{\mathrm{b}} \mathrm{TDB}$ deletion $=$ first intron sequence with deletion identified in T. aestivum NIL Triple Dirk B; intact = first intron sequence without TDB deletion

recorded (Table 1). 171 accessions of $T$. dicoccoides (Körn. ex Aschers. et Graebn.) Schweinf., T. ispahanicum Heslot and T. karamyschevii Nevski did not produce shoots without vernalization, thus confirming a winter growth habit (Additional file 1: Table S1).

\section{VRN-A1 promoter region variability}

Mutations within the promoter region appear to be responsible for the major differences between dominant and recessive alleles of the VRN1 gene and the cause of spring growth habit in wheat plants. Here, 57 accessions were screened using PCR amplification with A genome-specific primers designed by Yan et al. [20]. The PCR product of estimated length ( 700 bp) was obtained for all species studied. After cloning of PCR products, all clones obtained were sequenced and analyzed. In total, 69 sequences of the $V R N-A 1$ promoter region were identified. New sequences were aligned together with known recessive and dominant allelic variants of the VRN-A1 promoter region in di-, tetraand hexaploid wheat obtained from GenBank. Comparative analyses demonstrated that the most variable zone of the $V R N-A 1$ promoter is located in the region from -63 to 220 bp (Fig. 1).

Nine known allelic variants of the $V R N-A 1$ promoter Vrn-A1a.3, Vrn-A1b, Vrn-A1b.2, Vrn-A1b.6, Vrn-A1d, Vrn$A 1 e, V r n-A 1 f$, vrn-A1b.4, and vrn-A1 were identified. Promoter sequences of $V R N-A 1$ from 17 accessions of $T$. aethiopicum Jakubz., T. dicoccoides, T. dicoccum, T. durum
Desf. and T. turgidum L. were identical to the recessive allele $v r n-A 1$ identified in polyploids and $v r n-A 1 u$ of $T$. urartu (Table 1, Fig. 1). Four accessions T. aethiopicum K19059, T. turanicum Jakubz. K-31693, T. turgidum cv. Blancal (K-20416) and T. turgidum K-11597 contained one nucleotide substitution in the promoter when compared to the $v r n-A 1$ sequence (GQ451819).

The other 36 accessions had mutations in the promoter region distinguishing them from the recessive allelic variant $v r n-A 1$. The promoter region of the $V R N-A 1$ gene in $T$. dicoccoides $\mathrm{K}-15900$ and $\mathrm{i}$ : BS1E was identical to the Vrn-A1a.3 allele, and had an insertion of $231 \mathrm{bp}$ in length (Table 1, Fig. 1). Five variants of $V r n-A 1 b$ allele were identified in accessions $T$. dicoccum cv. Dichter Rotlicher (K-1730), T. durum K13768, T. turgidum K-3047 and K-13489, T. polonicum K-19597 and K-43335. All sequences of Vrn-A1b promoter had common mutation which distinguish them from $v r n-A 1$ sequence but vary in the length and sequences of VRN-box (Table 1, Fig. 1). Sequences of $T$. durum K-13768 and T. dicoccum cv. Dichter Rotlicher (K-1730) were identical to previously described variants $V r n-A 1 b .1$ and $V r n-A 1 b .2$, correspondingly. $V N R-A 1$ promoter sequences of $T$. turgidum K-13489 and K-3047, T. polonicum K-19597 and K-43335 were identical to each other and VrnA1b.6 allele identified in tetraploid and hexaploid wheat species [19]. 


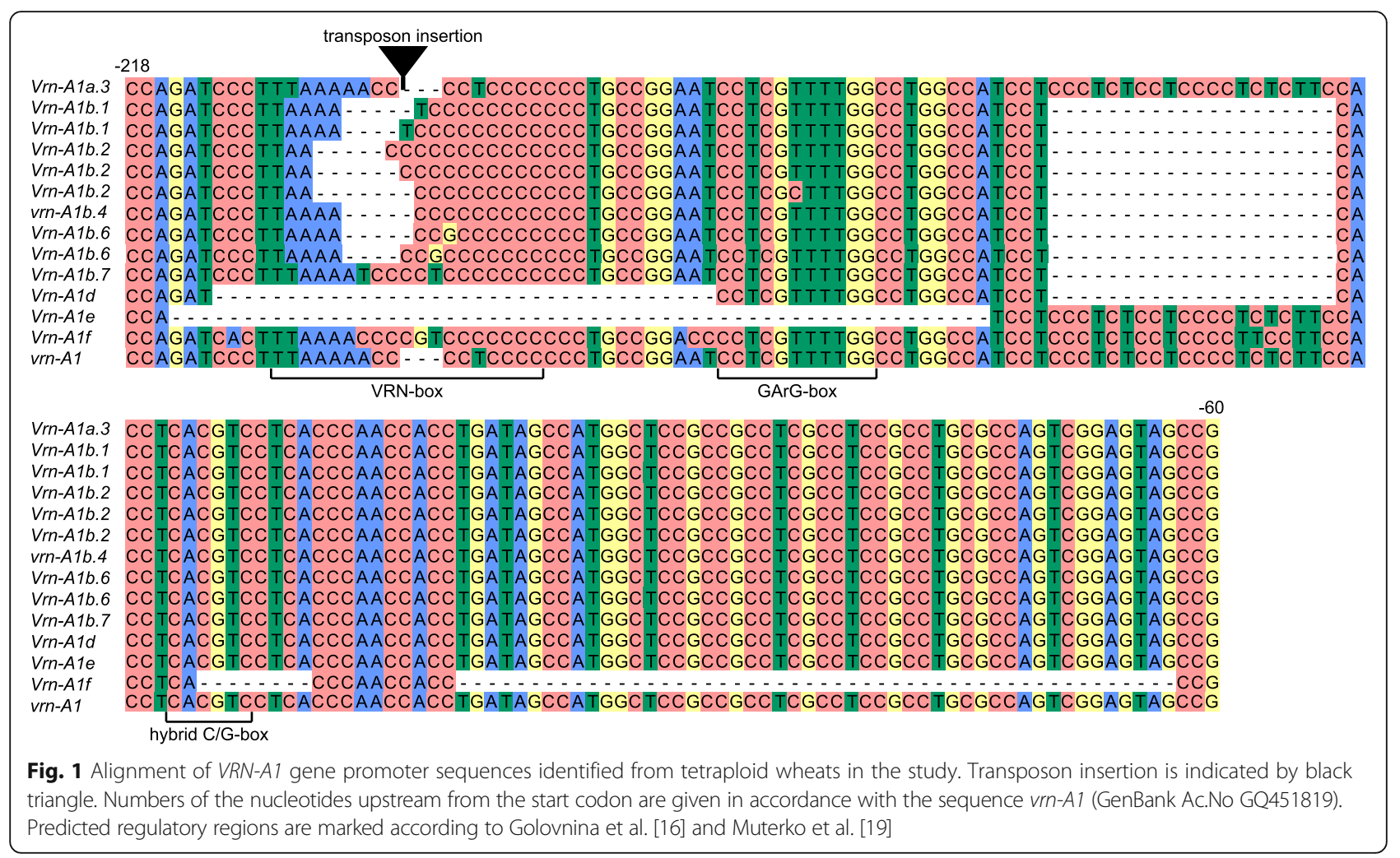

Five accessions of Dicoccoides section had two variants of the Vrn-A1 promoter sequence, both of which corresponded to the variants of $V r n-A 1 b$ allele (Table 1 ). Three accessions T. durum K-18118 and IG 85879, and T. polonicum L. K-17893 possess identical set of promoter sequences: $V r n-A 1 b .1$ variant identified previously and sequence differed from $\mathrm{Vrn}-\mathrm{A} 1 \mathrm{~b} .1$ only by insertion of one nucleotide "C" in C-rich segment of VRN-box (Fig. 1). Two sequences from accessions of $T$. dicoccoides IG346783 and T. dicoccum K-7500 represent Vrn-A1b.2 variant but vary in the length of C-rich segment (Fig. 1).

The T. timopheevii KU107-1, K-29540 and K-38555 had 8 and 50 bp deletions as in the Vrn-Alf sequence reported in T. araraticum (GQ451762) [16] (Table 1, Fig. 1). One accession of $T$. araraticum $\mathrm{K}-30234$ contained two sequences of the $V r n-A 1 f$ allele which varied in the length of the C-rich segment of VRN-box.

We observed a 54 bp deletion in the promoter region of T. carthlicum K-7106 that was identical to the VrnAle alleles of $T$. durum (GQ451821) and T. dicoccum (AY616463) (Table 1, Fig. 1).

Eleven accessions of $T$. dicoccoides had two different deletions one $20 \mathrm{bp}$ in length between -136 and -157 and another one $32 \mathrm{bp}$ in length between -179 and -212 nucleotides upstream of the start codon and were identical to the Vrn-A1d allele [17, 20] (Table 1, Fig. 1).

The most interesting were two groups of accessions which possessed two different variants of $\mathrm{Vrn}-\mathrm{Al}$ promoter sequences. First group of accession include $T$. dicoccoides PI428105, PI352324 and PI352328 and T. araraticum K58667 which are characterize by the presence of $\mathrm{Vrn}$-Alf reported in T. araraticum (GQ451762) and one of two variants $V r n-A 1 b$ allele (Table 1). Second promoter sequences identified in T. dicoccoides PI428105 and PI352328 were identical to recessive variant vrn-A1b.4. In case of the $T$. dicoccoides PI352324 and T. araraticum K-58667 $\mathrm{s}$ sequences differed from vrn-A1 by 20 bp deletion located $137 \mathrm{bp}$ upstream of the start codon, "A- > T" replacement and "CCC" insertion within the VRN-box. This variant was designated as Vrn-A1b.7 (Fig. 1). Second group of accessions include $T$. dicoccoides PI467027, PI467014 and PI467019 possess two sequences of VRN-A1 promoter: $V r n-A 1 d$ and Vrn-A1f alleles. Schematic representation of all VRN1 promoter sequences identified in di- and tetraploid species is presented in Additional file 2: Figure S1. Geographical distribution of the VRN-A1 alleles identified in the studied samples is presented on Additional file 2: Figure S2.

\section{VRN-A1 first intron variability}

First intron $V R N-A 1$ gene in tetraploid wheats was analyzed using three primer pairs (Additional file 1: Table S2). The first primer pair (Ex1/C/F and Intr1/A/R3) allowed us to amplify the sequences of the $V R N-A 1$ first intron which possesses a deletion of a characteristic length previously described for T. durum 'Langdon' [22]. The short PCR 
product ( 480 bp in length) containing the deletion was obtained for 7 accessions of T. aethiopicum (K19301, K-19398, K-19553, K-19253, K-19650, K-43766 and St56), 1 accession of T. dicoccoides (K-62328), 3 accessions of $T$. dicoccum (K-20749, K-1730 and K40306), 4 accessions of T. durum (K-17784, K-17787, K-52989 and cv. Langdon), 1 accession of T. polonicum (K-17893) and 2 accessions of T. turgidum (cv. Maiorka (K-16156) and cv. Zafrani (K-11597)) (Table 1). These sequences were identical to the $V r n-A 1 c$ allele of $T$. turgidum 'Langdon', possessing a 7222-bp deletion from 391 bp to 7612 bp compared to the T. aestivum NIL Triple Dirk C vrn-A1 allele (AY747600) [22].

No positive results of PCR amplification were obtained with the primers Intr1/A/F2 and Intr1/A/R3. Thus, no sequences of the $V R N-A 1$ first intron containing the deletion identified in hexaploid Afghanistan landrace IL369 were presented among analyzed species. The last primer pair (Intr1/C/F and Intr1/AB/R) allowed us to amplify the intact sequences of the first intron, producing a product of the expected length ( 1000 bp) for 39 tetraploid wheat accessions (Table 1).

\section{VRN-B1 and VRN-G1 promoter region variability}

The $B$ genome-specific primers were used to amplify and sequence the promoter region of five accessions of section Dicoccoides species, with recessive vrn-A1 promoter sequences and an intact $V R N-A 1$ first intron. The fragment of the expected length $\sim 1200 \mathrm{bp}$ was obtained for all analyzed species. Further comparative analyses demonstrated that almost all clones from accessions of Dicoccoides section contained sequences of the intact $v r n-B 1$ allele (Table 2). The only exception was $T$. dicoccoides IG46225, for which two different clones were identified. The first clone corresponded to the recessive $v r n-B 1$ alleles identified previously [16, 21, 22]. The second clone differed from the recessive allele by 29 nucleotide substitutions, one deletion and one insertion of a single nucleotide in the region from -220 to -155 bp upstream from the start codon (11\% of dissimilarity) (Table 2, Fig. 2, Additional file 2: Figure S1). The present allelic variant was different from all known B genome alleles of VRN1 gene and was named VrnB1dic. No VRN-B1 promoter sequences containing retrotransposon insertions, previously described by Chu et al. [21], were identified among analyzed accessions.

The VRN-G1 promoter was analyzed for five accessions of section Timopheevii species (Table 2). Sequences of the VRN-G1 promoter were identified for T. araraticum (accession K-30234) and T. timopheevii (accessions K-38555, K-29540 and K-29540). The 3' part of these sequences was identical to the sequence of Vrn-G1a allele from T. timopheevii K-38555 (GQ451755), while the 5' end was identified for the first time and differed from
vrn-G1 promoter by two deletions: 8 and 74 bp in length (Fig. 2; Additional file 2: Figure S1). The recessive vrnG1 promoter sequences were identified only for accession K-58667 of T. araraticum.

\section{VRN-B1 and VRN-G1 first intron variability}

First introns of $V R N-B 1$ and $V R N-G 1$ genes were analyzed using two primer pairs (see the Methods section). Sequences of the VRN-B1 first intron possessing the large deletion described for T. aestivum NIL Triple Dirk $B$ [22] were amplified with the primers Intr1/B/F and Intr1/B/R3. Positive results of the PCR amplification with this primer pair were obtained for one out of ten studied accessions (Table 2). The sequence of T. aethiopicum K-18999 differs from the first intron sequence of T. aestivum NIL Triple Dirk B (AY747603) by two nucleotide substitutions. The remaining 9 accessions gave positive results with the second primer pair (Intr1/B/F and Intr1/B/R4), and intact sequences of $V R N-B 1$ and $V R N-G 1$ first introns ( 1150 bp in length) were amplified (Table 2).

\section{Genetic control of growth habit in tetraploid species of Dicoccoides section}

Monogenic or digenic control of growth habit in some spring accessions of tetraploid wheat species of Dicoccoides section was analyzed in the genetic experiments. Accessions of tetraploid species were crossed with $T$. dicoccum cv. Black Winter Emmer (BWE) which has recessive $v r n-A 1$ and $v r n-B 1$ alleles and two nearisogenic tester lines, which are characterized with specific dominant $V r n-A 1$ (i: BS1E) or $V r n-B 1$ (i: BS2E) alleles. It was demonstrated that spring growth habit of six accessions of $T$. dicoccoides, $T$. dicoccum and $T$. durum is controlled by a single dominant gene VRN-A1 (Table 3). $\mathrm{F}_{2}$ hybrids of $T$. dicoccum cv. Dichter Rotlicher (K-1730), T. dicoccum cv. Bastard Emmer verastelter (K40306) and T. durum cv. Langdon, K-17784 and K17787 with BWE showed the segregation ration 3 to 1 , while their $F_{2}$ hybrids with BS1E showed no segregation and spring growth habit (Table 3). Thus, the results confirmed that the Vrn-Alc allele is dominant. For $T$. dicoccoides ICG №23 monogenic control of spring growth habit was shown (Table 3). All $\mathrm{F}_{2}$ hybrids of $T$. dicoccoides ICG №23 with BS1E showed spring growth habit which allows us to confirm that newly identified Vrn-A1d allele is dominant.

A correlation between certain dominant variant of $V R N-A 1$ genes and number of days to heading as well as a correlation between certain species and number of days to heading were not identified for the studied accessions of tetraploid wheats (Additional file 2: Figure S3, Figure S4). 


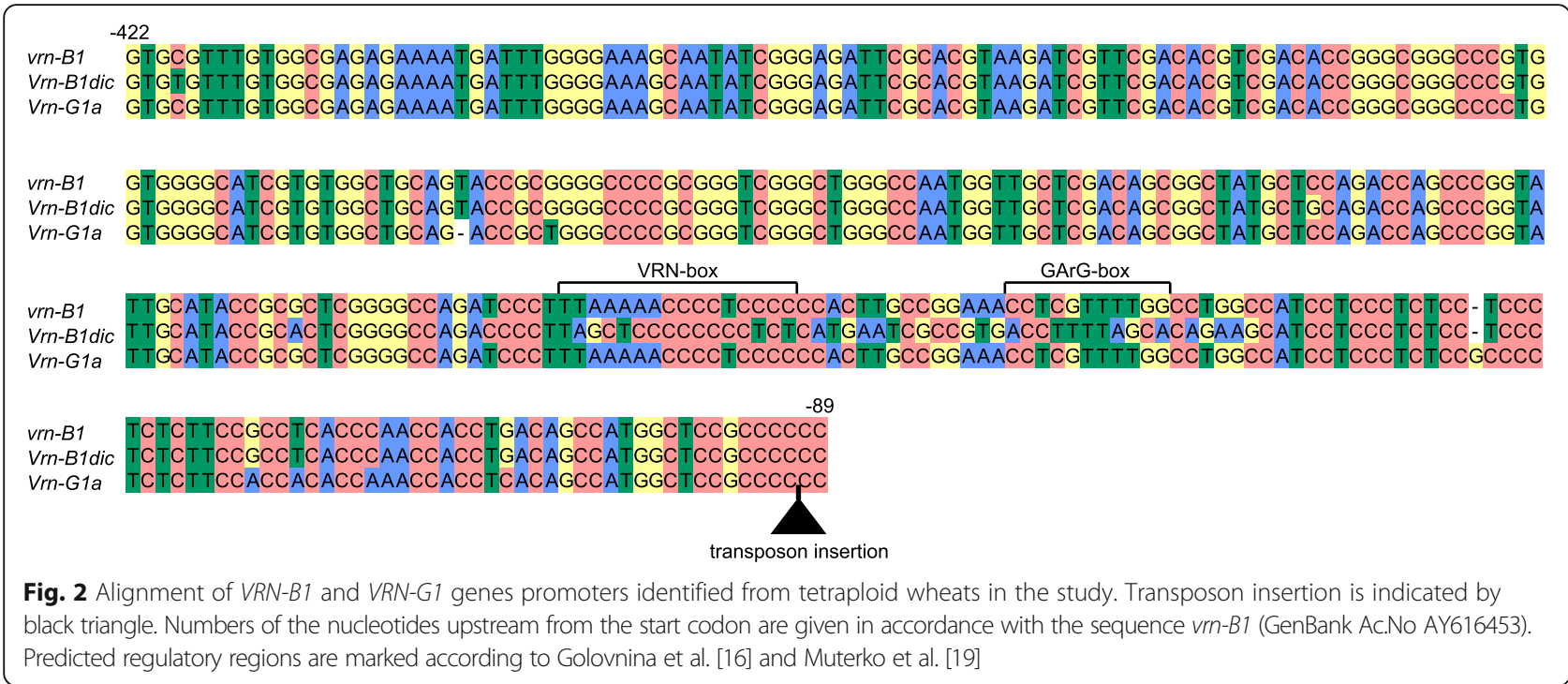

\section{Discussion}

\section{Predicted source of the spring growth habit among tetraploid wheat species}

Variability in the growth habit (spring vs. winter) of tetraploid wheat has been studied in greenhouse tests and 57 spring accessions were subsequently identified. For four of the species analyzed (T. polonicum, T. carthlicum, $T$. aephiopicum, and T. timopheevii) no winter accessions were identified in the present study nor in a previous study by Goncharov [36]. All studied accessions of T. ispahanicum Heslot and T. karamyschevii Nevski were found to have a winter growth habit. The following analysis of the VRN-A1,VRN-B1 and VRN-G1 genes in accessions with a spring growth habit revealed the presence of different mutations within the promoter or first intron region of those genes.

For 36 of the spring accessions studied, we identified variability within the promoter region of the VRN-A1 gene (Table 1). $V R N-A 1$ promoter sequences of these 36 accessions matched one of four different dominant alleles (Vrn-A1a.3, Vrn-A1d, Vrn-A1e, Vrn-A1f) or one of four $V r n-A 1 b$ allele variant (Vrn-A1b.1, Vrn-A1b.2, VrnA1b.6, Vrn-A1b.7). The presence of Vrn-A1a, Vrn-A1b.1, Vrn-A1b.2, Vrn-A1b.6, Vrn-A1d,Vrn-Ale or Vrn-Alf was previously predicted to be a determinant of the spring growth habit in wheat species $[16,17,19,20]$.

Seven of the 36 spring accessions studied possess two different allelic variants of $V R N-A 1$, at least one of which was dominant and could led to the spring growth habit. The presence of two different alleles in one accession could be explained by heterozygosity of the plant material or the variation of copy number of genes due to the duplication of the investigated region or the part of the genome. Presence of two different alleles has not been described for diploid or hexaploid wheat species, but this has been identified in the wild tetraploid species $[16,37]$.

Table $3 F_{2}$ segregation for growth habit in crosses of tetraploid wheat species with three control lines

\begin{tabular}{|c|c|c|c|c|c|}
\hline \multirow[t]{2}{*}{ Accession } & \multicolumn{3}{|c|}{$\begin{array}{l}\text { Segregation into spring vs. winter forms in the } \\
F_{2} \text { generation in crosses witha: }\end{array}$} & \multirow[t]{2}{*}{$\begin{array}{l}\text { Genotype identified by } \\
\text { hybrodological method }\end{array}$} & \multirow[t]{2}{*}{$\begin{array}{l}\text { VRN-A1 allele identified by } \\
\text { molecular methods (promoter/intron1) }\end{array}$} \\
\hline & $\begin{array}{l}\text { BWE } \\
\text { (vrn-A1 vrn-B1) }\end{array}$ & $\begin{array}{l}\text { BS1E } \\
(\text { Vrn-A1 vrn-B1) }\end{array}$ & $\begin{array}{l}\text { BS2E (vrn-A1 } \\
\text { Vrn-B1) }\end{array}$ & & \\
\hline $\begin{array}{l}\text { T. dicoccoides (Körn. ex Aschers. et } \\
\text { Graebn.) Schweinf. ICG №23 }\end{array}$ & NA & $22: 0$ & $95: 8^{b}$ & Vrn-A1 vrn-B1 & $\begin{array}{l}\text { Vrn-Ald/no Langdon and IL369 } \\
\text { deletions }\end{array}$ \\
\hline $\begin{array}{l}\text { T. dicoccum (Schrank) Schuebl. cv. } \\
\text { Dichter Rotlicher K-1730 }\end{array}$ & $77: 22^{c}$ & $156: 0$ & NA & Vrn-A1 vrn-B1 & Vrn-A1b.2/Vrn-A1c \\
\hline $\begin{array}{l}\text { T. dicoccum cv. Bastard Emmer } \\
\text { verastelter K-40306 }\end{array}$ & $214: 93^{c}$ & 114:0 & NA & Vrn-A1 vrn-B1 & $v r n-A 1 / V r n-A 1 c$ \\
\hline T. durum Desf. cv. Langdon & $203: 40^{c}$ & 102:0 & NA & Vrn-A1 vrn-B1 & $v r n-A 1 / V r n-A 1 c$ \\
\hline T. durum Desf. K-17784 & $183: 63^{c}$ & 113:0 & NA & Vrn-A1 vrn-B1 & vrn-A1/Vrn-A1C \\
\hline T. durum Desf. K-17787 & $63: 14^{c}$ & 113:0 & NA & Vrn-A1 vrn-B1 & $v r n-A 1 / V r n-A 1 c$ \\
\hline
\end{tabular}

aplants thatT before $F_{1}$ hybrids of $\mathrm{i}$ BS2E and BWE were classified as spring, whereas plants that remained in the vegetative phase were classified as winter bdigenic control, $X^{2}$ value for ration is not higher than 3.84

'monogenic control, $X^{2}$ value for ration is not higher than 3.84 
Allele vrn-A1 and variants of $V r n-A 1 b$ were the most frequently occurring, and were identified for 21 and 15 spring tetraploid accessions, respectively (Table 1). Vrn$A 1 d$ and Vrn-Alf were also common and presented in 14 and 11 spring tetraploid accessions, respectively. Dominant inheritance of Vrn-Ald was confirmed in the genetic experiments. The remaining two alleles are rare, the $V r n-A 1 a$ was identified in two accessions while VrnAle was found only once (Table 1 ).

21 accessions of T. aethiopicum, T. dicoccoides, T. dicoccum, T. durum, T. polonicum, T. turanicum and T. turgidum contained the recessive allele of the VRN-A1 promoter. Therefore, its spring growth habit could be explained by other changes in the VRN1 gene sequences, this may include mutations in the $V R N-B 1$ promoter regions, as well as in the first intron sequence of both VRN-A1 and $V R N-B 1$ genes. 16 out of 21 accessions showed the presence of a large deletion within the VRN-A1 first intron region $(V r n-A 1 c)$ and dominant inheritance of this allele was confirmed in the genetic experiments (Table 1, Table 3). Only one of the studied accessions possessed a disruption within the $V R N-B 1$ promoter sequence. $T$. dicoccoides IG46225 contains two different $V R N-B 1$ promoters: the first one was identical to the intact sequence of the $v r n-B 1$ allele, whereas the second one displayed a new allelic variant, named Vrn-B1dic. The presence of the Vrn-B1dic allele could be the cause of spring growth habit in T. dicoccoides IG46225. Vrn-B1dic allele is characterized by unexpected high dissimilarity in compare to the $v r n-B 1$ allele. If we exclude the deletions and insertions cases the other dominant alleles of VNR1 genes of di- and polyploidy wheats differ from recessive alleles by several SNPs [19, 22, 23, 27, 29].
Alternatively, we could suggest that the Vrn-B1dic represent the pseudogene copy originated by duplication within one genome. Previously, the investigation of the bread wheat genome showed the major impact of single gene duplications on the wheat evolution [38]. Moreover the gene duplications followed by gene loss, subfunctionalization or neofunctionalization played significant role in the evolution of MADS-box transcription factors [39]. Investigation of the Vrn-B1dic expression is required to proof the hypothesis.

T. aethiopicum K-18999 was the only accession for which we identified intact promoters of $V R N-A 1$ and $V R N-B 1$ genes, and an intact first intron of the VRN-A1 gene. A deletion has been found in the first intron of the $V R N-B 1$ gene. This deletion is a predicted cause of $T$. aethiopicum K-18999's spring growth habit.

One group of accessions is of particular interest in the investigation of the possible cause of spring growth habit in tetraploid wheat species. T. dicoccum cv. Dichter Rotlicher (K-1730) and T. polonicum K-17893, both of which posses the dominant allele of the $V R N-A 1$ promoter, but contain deletions in the first introns of the VRN-A1 gene. Five accessions of section Timopheevii had dominant alleles of both VRN-A1 and VRN-G1 promoters. Both variants could contribute to the formation of spring growth habit.

Thus, the predicted source of spring growth habit was determined for 54 of 57 tested accessions, including those described previously as well as the novel disruption identified in the promoter or first intron sequences of $V R N-A 1$, $V R N-B 1$ and/or $V R N-G 1$ genes. The rest of the accessions, which include T. aethiopicum K-19059, T. turanicum K31693 and T. turgidum cv. Blancal (K-20416), contained

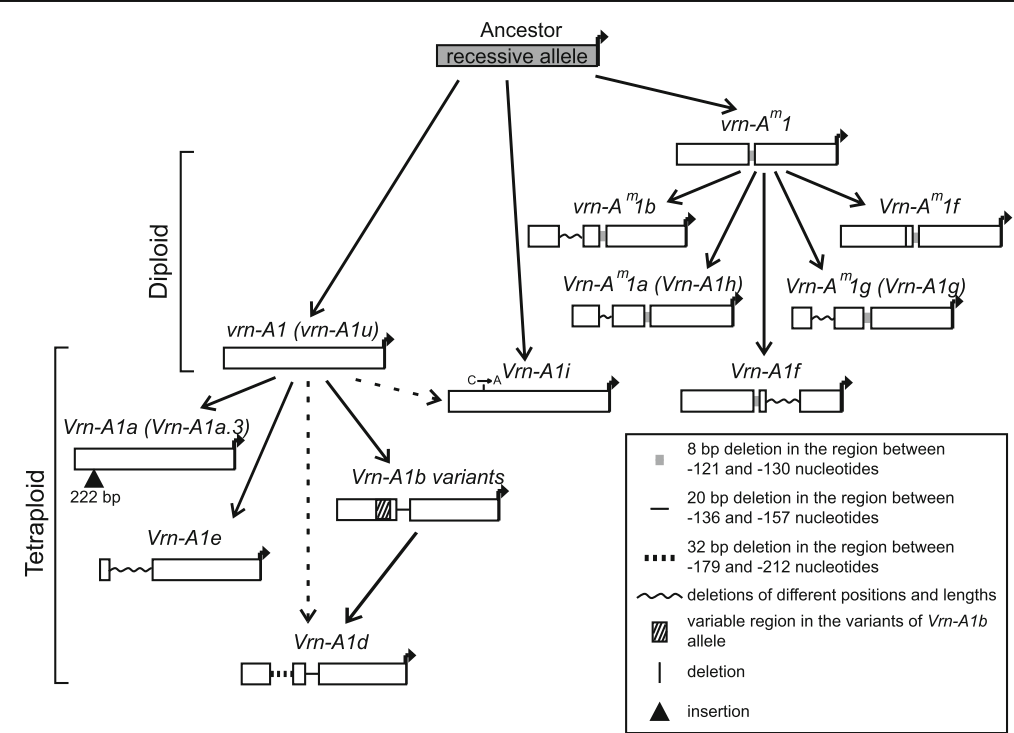

Fig. 3 Scheme of the evolution of VRN-A1 promoter sequences from di- and tetraploid wheat. Arrows indicate the ways of sequences evolution, dotted arrows indicate the alternative ways 
the recessive $V R N-A 1$ and $V R N-B 1$ promoter sequences and an intact first intron of both $V R N-A 1$ and $V R N-B 1$ genes. A cause for the spring growth habit in these accessions remains unknown.

\section{Origin of $V R N-A 1$ promoter variability in tetraploid wheat species}

The variability identified in this study is probably the direct cause of the differences between spring and winter growth habit in tetraploid wheat species. To date, 20 different alleles of the $V R N-A 1$ promoter were identified in the genomes of $\mathrm{di}$ - and tetraploid species (Fig. 3; Additional file 2: Figure S1). Three recessive alleles differing by short deletions are presented in diploids, while the remaining four dominant alleles possess different substitutions, deletions and insertions compared to the recessive alleles. Three recessive and eleven dominant are presented among tetraploid accessions (Figs. 3 and 4).

Analysis of the sequences allowed us to suggest the predicted pattern of evolution of $V R N-A 1$ promoters. The recessive allele $v r n-A 1$ identified in tetraploid species was inherited from diploids, presumably from $T$. urartu (Figs. 3 and 4).

Dominant alleles Vrn-A1a (Vrn-A1a.3), Vrn-A1e, Vrn-A1i and variants of $V r n-A 1 b$ first appeared in tetraploid species and apparently originated from $v r n-A 1$ by way of short deletions in the case of $V r n-A 1 b$ and $V r n-A 1 e$, substitution in case of $V r n-A 1 i$ and insertions for $V r n-A 1 a .3$ (Fig. 3). The variant of $V r n-A 1 b$ allele except $V r n-A 1 b .7$ and $V r n-A 1 e$ are presented only in tetraploid species of the Dicoccoides section, and may originate from the vrn-A1 allele after sections separation. Allele $V r n-A 1 b .7$ is presented in both Dicoccoides and Timopheevii sections and apparently originated in a common tetraploid

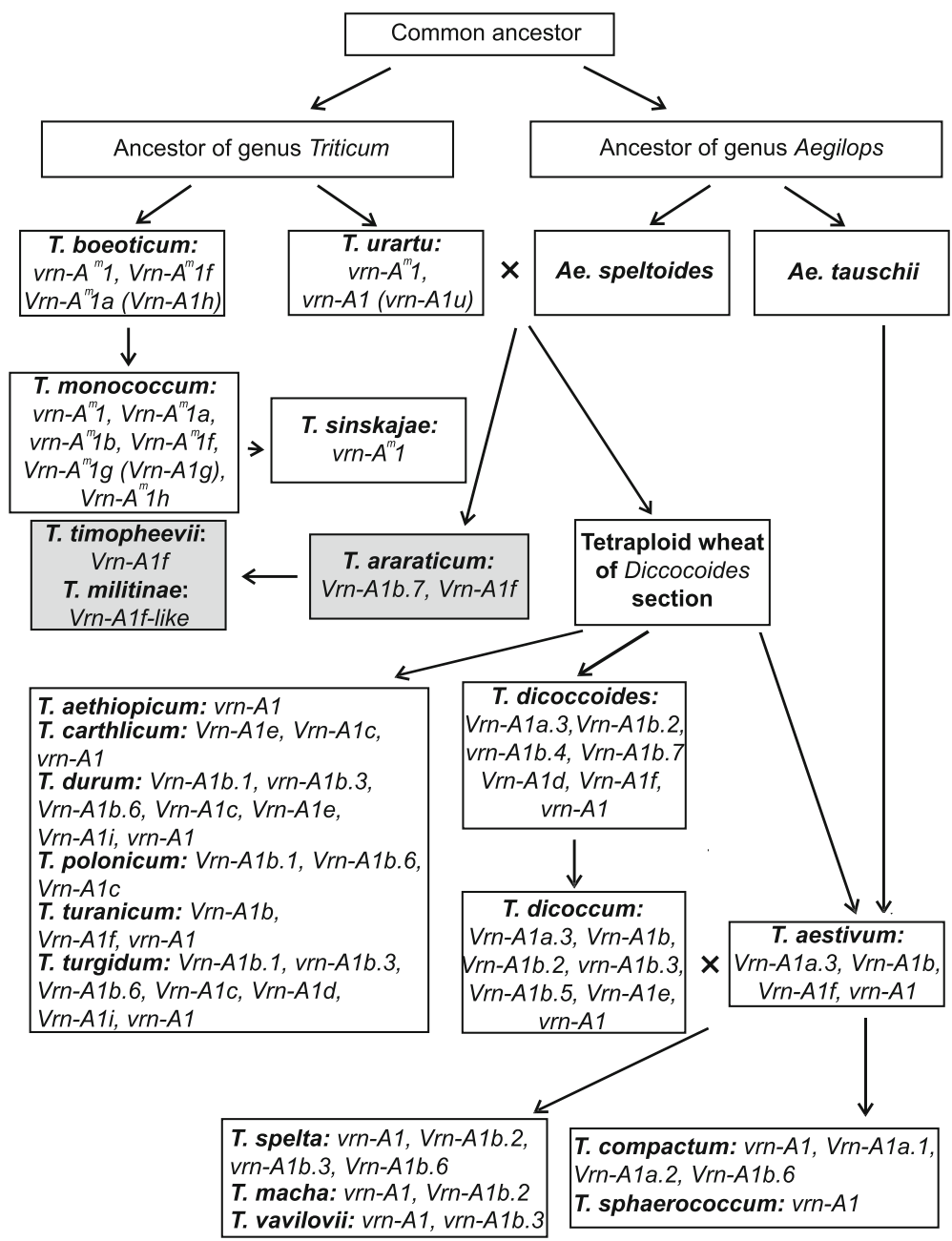

Fig. 4 Scheme of Triticum and Aegilops genera evolution (according to Goncharov [41], with additions). Different alleles of VRN-A1 gene among wheat species are presented in appropriate boxes next to the species names. Section Timopheeevii is presented in grey boxes, while section Monococcon, Dicoccoides and Triticum are in white boxes 
wheat ancestor before sections separation (Fig. 4). Among tetraploids the distribution of the Vrn-Ala.3 allele was restricted by $T$. dicoccum and $T$. dicoccoides accessions of the Dicoccoides section (Fig. 4).

The dominant Vrn-A1d allele is presented in both Dicoccoides and Timopheevii sections, and may originate from one of the $V r n-A 1 b$ variants through extension of the deletion. Alternatively, the formation of two deletions in the vrn-A1 allele could give the Vrn-A1d (Fig. 3). Allele $V r n-A 1 d$ probably originated only once in the ancestor of tetraploid wheat species and evidently was not inherited by hexaploid wheat species (Figs. 3 and 4). Kato et al. [40] predicted that spring accessions of $T$. dicoccoides evolved from a winter forms as an adaptation to warmer conditions. However in the present investigation no correlation between the presence of Vrn-A1d allele and particular environmental conditions of collection sites of studied accessions was identified (Table 1).

The most interesting case is the Vrn-Alf allele, which in comparison to other dominant alleles of tetraploid wheat, originated from the recessive $v r n-A^{m} 1$ allele of a common ancestor of diploid wheat species (Fig. 3). Vrn$A 1 f$ allele is on a par with dominant alleles of diploids obtained by deletion in the recessive $v r n-A^{m} 1$ allele promoter. To date, the Vrn-A1f allele has been identified in diploids (T. monococcum, T. urartu, T. boeoticum), tetraploids (Dicoccoides and Timopheevii sections) and hexaploids (T. aestivum) (Fig. 4) [16, 17].

\section{Conclusions}

The growth habit was investigated for 228 accessions of 12 tetraploid wheat species. The promoter and first intron regions of VRN1 genes were analyzed in 57 spring accessions of 10 tetraploid species. Comparative analysis revealed the novel allele of VRN-A1 (Vrn-A1b.7) and VRN-B1 (Vrn-B1dic). Vrn-A1d was widely distributed across the accessions of $T$. dicoccoides. In the genetic experiments the dominant mode of inheritance was shown for the Vrn-A1d and Vrn-A1c alleles. It is assumed that the presence of $V r n-A 1 d$ allele is associated with the formation of spring growth habit in the 11 accessions of $T$. dicoccoides. Vrn-B1dic is a unique allele characterized by the unexpected high level of promoter sequence dissimilarity in comparison to the vrn-B1. This allele was identified in the only accession of $T$. dicoccoides (IG46225) and further investigations are required to determine the role of this allele in the formation of spring growth habit. Novel allelic variants identified in the represent study provide a useful resource for fundamental investigations and could be used in agricultural production to expand the biodiversity of cultivated of wheat species. The summarization of the results regarding to the VRN1 alleles identified to date in di- and polyploid wheat species allowed us to discuss the evolution of the alleles.

\section{Additional files}

Additional file 1: Table S1. List of tetraploid wheat species used in the study and their growth habit. Species names are given according to Dorofeev et al. [1] and Goncharov [2]. Table S2. Set of primers used in the present study. (PDF $444 \mathrm{~kb}$ )

Additional file 2: Figure S1. Schematic representation of VRN-A1 (A), VRN$B 1$ and VRN-G1 (B) promoter region variability identified in di- and tetraploid wheat species. Insertions are indicated by black triangles, insertion lengths are marked under the black triangles. Deletions are indicated by dotted black lines. Hatched rectangles indicate variable region in the variants of V rn-A1b allele. Locations of the deletions and insertions are marked in base pair numbers upstream from the start codon, in accordance with the sequence vrn-A1u (GenBank Ac.No GQ451819). * - allele was identified by Golovnina et al. 2010. Figure S2. Geographical distribution of different variants of VRN-A1 gene from accessions of studied tetraploid wheat species. Abbrevations: a Vrn-A1a.3; b - variants of Vrn-A1b; d - Vrn-A1d; e - Vrn-Ale; f - Vrn-A1f; u - vrn-Al (vrn-A1u) allele. Numbers on the right of the graphic symbols match the numbers of studied wheat accessions which possess certain allelic variant. Figure S3. Distribution of heading time among studied accessions of ten tetraploid wheat species. Different species are marked by different colors. Figure S4. Distribution of heading time among VRN-A1 alleles identified in studied accessions of ten tetraploid wheat species. Different allelic variants are marked by different colors. (PDF 3302 kb)

\section{Acknowledgments}

We thank Ms. Carly Schramm, School of Biological Sciences, Flinders University (Australia), for critical review of our manuscript.

\section{Declarations}

This article has been published as part of BMC Plant Biology Volume 16 Supplement 3, 2016: Selected articles from BGRSISB-2016: plant biology. The full contents of the supplement are available online at http://bmcplantbiol.biomed central.com/articles/supplements/volume-16-supplement-3.

\section{Funding}

Funding for this work was provided by the RAS project number 21 (grant number: 0324-2015-0009) and the Russian Foundation for Basic Research (Grant No. 16-34-00688). Publication of this article has been funded by the Russian Foundation for Basic Research (Grant No. 16-34-00688).

\section{Availability of data and material}

The VRN1 gene sequences obtained in this study are available in GenBank, with the accession numbers KP063935-KP063977, KP063980-KP063992, KP063995-KP064001, KP064003-KP064012, KP260494, KP260496, KP260497, KR055675-KR055677, KR055679, KR055680, KR055683, KR055684, KR055686, KR055688, KR055694, KR055695, KR055697, KR055698.

\section{Authors' contributions}

IK analyzed the VRN1 genes sequences and wrote the manuscript. W cloned and sequenced VRN1 genes and contributed to the preparation of the manuscript. EYaK carried out the greenhouse experiments. AB conceived and designed the molecular genetics experiments, revised the manuscript. NPG designed the greenhouse experiments, analyzed the results, contributed to the discussion and participated in preparing the manuscript. All authors read and approved the final manuscript.

\section{Competing interests}

The authors declare that they have no competing interests.

\section{Consent for publication}

Not applicable.

\section{Ethics approval and consent to participate}

Not applicable.

\section{Author details}

${ }^{1}$ The Federal Research Center Institute of Cytology and Genetics SB RAS, Prospekt Lavrentyeva, 10, Novosibirsk 630090, Russian Federation.

${ }^{2}$ Novosibirsk State University, Pirogova 2, 630090 Novosibirsk, Russian 
Federation. ${ }^{3}$ Novosibirsk State Agrarian University, Dobrolubov Str., 160, Novosibirsk 630039, Russian Federation.

\section{Published: 16 November 2016}

\section{References}

1. Cockram J, Jones H, Leigh FJ, O'Sullivan D, Powell W, Laurie DA, Greenland AJ. Control of flowering time in temperate cereals: Genes, domestication, and sustainable productivity. J Exp Bot. 2007;58(6):1231-44.

2. Distelfeld A, Li C, Dubcovsky J. Regulation of flowering in temperate cereals. Curr Opin Plant Biol. 2009;12(2):178-84.

3. Yan L, Loukoianov A, Tranquilli G, Helguera M, Fahima T, Dubcovsky J. Positional cloning of the wheat vernalization gene VRN1. Proc Natl Acad Sci U S A. 2003;100:6263-8.

4. Yan L, Loukoianov A, Blechl A, Tranquilli G, Ramakrishna W, SanMiguel P Bennetzen JL, Echenique V, Dubcovsky J. The wheat VRN2 gene is a flowering repressor down-regulated by vernalization. Science. 2004; 303(5664):1640-4.

5. Yan L, Fu D, Li C, Blechl A, Tranquilli G, Bonafede M, Sanchez A, Valarik M, Yasuda S, Dubcovsky J. The wheat and barley vernalization gene VRN3 is an orthologue of FT. Proc Natl Acad Sci. 2006;103(51):19581-6.

6. Kippes N, Debernardi JM, Vasquez-Gross HA, Akpinar BA, Budak H, Kato K, Chao S, Akhunov E, Dubcovsky J. Identification of the VERNALIZATION 4 gene reveals the origin of spring growth habit in ancient wheats from South Asia. Proc Natl Acad Sci U S A. 2015;112(39):E5401-10.

7. Trevaskis B, Hemming MN, Dennis ES, Peacock WJ. The molecular basis of vernalization-induced flowering in cereals. Trends Plant Sci. 2007;12(8):352-7.

8. Fjellheim S, Boden S, Trevaskis B. The role of seasonal flowering responses in adaptation of grasses to temperate climates. Front Plant Sci. 2014;5:431.

9. Law CN, Worland AJ, Giorgi B. The Genetic Control of Ear-Emergence Time by Chromosomes 5A an 5D of Wheat. Heredity (Edinb). 1976;36(1):49-58.

10. Dubcovsky J, Lijavetzky D, Appendino L, Tranquilli G. Comparative RFLP mapping of Triticum monococcum genes controlling vernalization requirement. Theor Appl Genet. 1998;97(5-6):968-75.

11. Barrett B, Bayram M, Kidwell K. Identifying AFLP and microsatellite markers for vernalization response gene $V r n-B 1$ in hexaploid wheat using reciprocal mapping populations. Plant Breed. 2002;121(5):400-6.

12. Iwaki K, Nishida J, Yanagisawa T, Yoshida H, Kato K. Genetic analysis of Vrn-B1 for vernalization requirement by using linked dCAPS markers in bread wheat (Triticum aestivum L.). Theor Appl Genet. 2002;104(4):571-6.

13. Danyluk J, Kane NA, Breton G, Limin AE, Fowler BD, Sarhan F. TaVRT-1, a Putative Transcription Factor Associated with Vegetative to Reproductive Transition in Cereals. Plant Physiol. 2015;132:1849-60.

14. Murai K, Miyamae M, Kato H, Takumi S, Ogihara Y. WAP1, a Wheat APETALA1 Homolog, Plays a Central Role in the Phase Transition from Vegetative to Reproductive Growth. Plant Cell Physiol. 2003;44(12):1255-65.

15. Dubcovsky J, Loukoianov A, Fu D, Valarik M, Sanchez A, Yan L. Effect of photoperiod on the regulation of wheat vernalization genes VRN1 and VRN2. Plant Mol Biol. 2006;60(4):469-80.

16. Golovnina KA, Kondratenko EY, Blinov AG, Goncharov NP. Molecular characterization of vernalization loci VRN1 in wild and cultivated wheats. BMC Plant Biol. 2010;10:168.

17. Shcherban AB, Strygina KV, Salina EA. VRN-1 gene- associated prerequisites of spring growth habit in wild tetraploid wheat $T$. dicoccoides and the diploid A genome species. BMC Plant Biol. 2015;15(1):94.

18. Pidal B, Yan L, Fu D, Zhang F, Tranquilli G, Dubcovsky J. The CArG-box located upstream from the transcriptional start of wheat vernalization gene VRN1 is not necessary for the vernalization response. J Hered. 2009;100(3):355-64

19. Muterko A, Kalendar R, Salina E. Novel alleles of the VERNALIZATION1 genes in wheat are associated with modulation of DNA curvature and flexibility in the promoter region. BMC Plant Biol. 2016;16 Suppl 1:65-81.

20. Yan L, Helguera M, Kato K, Fukuyama S, Sherman J, Dubcovsky J. Allelic variation at the VRN-1 promoter region in polyploid wheat. Theor Appl Genet. 2004;109(8):1677-86.

21. Chu C, Tan C, Yu G, Zhong S, Xu SS, Yan L. A Novel Retrotransposon Inserted in the Dominant Vrn-B1 Allele Confers Spring Growth Habit in Tetraploid Wheat (Triticum turgidum L.). G3 (Bethesda). 2011;1(7):637-45.

22. Fu D, Szucs P, Yan L, Helquera M, Skinner JS, von Zitzewitz J, Hayes PM, Dubcovsky J. Large deletions within the first intron in VRN-1 are associated with spring growth habit in barley and wheat. Mol Genet Genomics. 2005; 273(1):54-65.

23. Ivaničová Z, Jakobson I, Reis D, Safar J, Milec Z, Abrouk M, Doležel J, Järve K, Valárik M. Characterization of new allele influencing flowering time in bread wheat introgressed from Triticum militinae. N Biotechnol. 2016;33(5 Pt B):718-27.

24. Shcherban $A B$, Efremova $T$, Salina EA. Identification of a new Vrn-B1 allele using two near-isogenic wheat lines with difference in heading time. Mol Breed. 2012;29(3):675-85.

25. Shcherban AB, Khlestkina EK, Efremova TT, Salina EA. The effect of two differentially expressed wheat VRN-B1 alleles on the heading time is associated with structural variation in the first intron. Genetica. 2013; 141(4):133-41.

26. Milec Z, Sumikova T, Tomkova L, Pankova K. Distribution of different Vrn-B1 alleles in hexaploid spring wheat germplasm. Euphytica. 2013;192(3):371-8.

27. Santra DK, Santra M, Allan RE, Campbell KG, Kidwell KK. Genetic and Molecular Characterization of Vernalization Genes Vrn-A1, Vrn-B1, and Vrn-D1 in Spring Wheat Germplasm from the Pacific Northwest Region of the U.S.A. Plant Breed. 2009;128(6):576-84.

28. Muterko A, Balashova I, Cockram J, Kalendar R, Sivolap Y. The New Wheat Vernalization Response Allele Vrn-D1s is caused by DNA Transposon Insertion in the First Intron. Plant Mol Biol Report. 2015;33(2):294-303.

29. Zhang X, Gao M, Wang S, Chen F, Cui D. Allelic variation at the vernalization and photoperiod sensitivity loci in Chinese winter wheat cultivars (Triticum aestivum L.). Front Plant Sci. 2015;6:470.

30. Zhang J, Wang Y, Wu S, Yang J, Liu H, Zhou Y. A single nucleotide polymorphism at the Vrn-D1 promoter region in common wheat is associated with vernalization response. Theor Appl Genet. 2012;125(8):1697-704.

31. Goncharov NP. Genetics of Growth Habit (Spring vs. Winter) in Tetraploid Wheats: Production and Analysis of Near-Isogenic Lines. Hereditas. 1999; 130(2):125-30.

32. Pugsley A. A genetic analysis of the spring-winter habit of growth in wheat. Aust J Agric Res. 1971;22:21.

33. Goncharov NP. Response to vernalization in wheat: its quantitative or qualitative nature. Cereal Res Commun. 2004;32(3):323-30.

34. Lu G, Moriyama EN. Vector NTI, a balanced all-in-one sequence analysis suite. Brief Bioinform. 2004;5(4):378-88.

35. Okonechnikov K, Golosova O, Fursov M, Varlamov A, Vaskin Y, Efremov I, Grehov G, Kandrov D, Rasputin K, Syabro M, Tleukenov T. Unipro UGENE: A unified bioinformatics toolkit. Bioinformatics. 2012;28(8):1166-7.

36. Goncharov NP. Genetic resources of wheat related species: the Vrn genes controlling growth habit (spring vs winter). Euphytica. 1998;100:371-6.

37. Chhuneja P, Arora JK, Kaur P, Kaur S, Singh K. Characterization of wild emmer wheat Triticum dicoccoides germplasm for vernalization alleles. J Plant Biochem Biotechnol. 2015;24(2):249-53.

38. Glover NM, Daron J, Pingault L, Vandepoele K, Paux E, Feuillet C, Choulet F. Small-scale gene duplications played a major role in the recent evolution of wheat chromosome 3B. Genome Biol. 2015;16:188.

39. Airoldi CA, Davies B. Gene duplication and the evolution of plant MADS-box transcription factors. J Genet Genomics. 2012;39(4):157-65.

40. Kato K, Mori Y, Beiles A, Nevo E. Geographical variation in heading traits in wild emmer wheat, Triticum dicoccoides. I. Variation in vernalization response and ecological differentiation. Theor Appl Genet. 1997;95:546-52.

41. Goncharov NP. Genus Triticum L. taxonomy: The present and the future. Plant Syst Evol. 2011;295(1):1-11.

42. Dorofeev VF, Filatenko AA, Migushova FF, Udachin RA, Jakubtsiner MM, Cultivated Flora of the USSR. Vol. 1. Pshenitsa (Wheat). Leningrad: Kolos; 1979 (In Russian). 